\title{
Prevalence of high risk human papilloma viruses 16 and I8 (HR-HPVI6\&I8) among Sudanese patients with esophageal cancer
}

\begin{abstract}
Objective: The aim of this study was to screen for the presence of High Risk Human Papilloma Viruses 16 and 18 (HR-HPV16\&18) among Sudanese patients with esophageal cancer.

Methodology: The study was retrospectively examined 100 formalin fixed paraffin wax embedded tissue's blocks for the presence of HR-HPV subtypes 16 and 18 by Immunohistochemical methods. All samples were previously obtained from Sudanese patients with esophageal cancer.

Results: Regarding IHC staining for HPV 16 and 18, positive findings were revealed in 13 $(13 \%)$ and couldn't be disclosed in $88(87 \%)$ of the study subjects, hence, none of the cases was identified with HPV18.

Conclusion: HR-HPV subtype 16 is strongly contributed to the etiology of esophageal carcinoma in Sudan. Urgent preventive strategies are highly recommended to reduce the burden of esophageal cancer in Sudan.
\end{abstract}

Keywords: esophageal cancer, Sudan, hpv16, hpv18
Volume 5 Issue 6 - 2016

\author{
Hussain Gadelkarim Ahmed, 1,2 Mohmad \\ Ahmad Babikir lbraheem Beely, ${ }^{3}$ Mohammed \\ Siddig Abd El Aziz, ${ }^{4}$ Fawaz D Alshammari, ${ }^{5}$ \\ Ahmed Abdalla Agab Eldour ${ }^{3}$ \\ 'Department of Pathology, University of Hail, KSA \\ ${ }^{2}$ Department of Histopathology and Cytology, University of \\ Khartoum, Sudan \\ ${ }^{3}$ Department of Medical Laboratory, University of Kordofan, \\ Sudan \\ ${ }^{4}$ Department of Histopathology and Cytology, Sudan University \\ for Science and Technology, Sudan \\ ${ }^{5}$ Department of Medical Lab Science, University of Hail, KSA
}

Correspondence: Hussain Gadelkarim Ahmed, Department of Pathology, College of Medicine, University of Hail, 2440, Kingdom of Saudi Arabia, Email hussaingad 1972@yahoo.com

Received: August 31, 2016 | Published: September 21, 2016

\section{Introduction}

Esophageal cancer, usually involve esophageal squamous cell carcinoma (SCC) and esophageal adenocarcinoma (AC), is one of the most common malignant tumors in the world. ${ }^{1}$ Esophageal cancer is the sixth leading cause of cancer death in the world. ${ }^{2}$ Esophageal squamous-cell carcinoma and esophageal adenocarcinoma represent the core histological subtypes of esophagus cancer. ${ }^{3}$ Esophageal carcinoma represents a poor prognosis among gastrointestinal cancers. While esophageal squamous cell carcinoma predominates worldwide, Western nations have seen a marked rise in the incidence of esophageal adenocarcinoma that parallels the obesity epidemic. Efforts directed toward early detection have been difficult, assumed that dysplasia and early cancer is generally asymptomatic. However, significant developments have been made in the past 10 to 15 years that permit for endoscopic management and often cure in early stage esophageal malignancy. ${ }^{4}$

Many factors have been incriminated to the etiology of esophageal cancer. The carcinogenesis of esophageal AC is initiated by the acid exposure of the esophageal mucosa from stomach ${ }^{5}$ whereas that of the esophageal SCC is linked to the chronic irritation of carcinogens mainly by the cigarette smoking, ${ }^{6}$ alcohol $^{7}$ and hot beverage. ${ }^{8} \mathrm{~A}$ potential role of HPV in the rise of esophageal AC was proposed recently. Multiple meta-analyses support the outcome of a higher HPV detection rate in regions associated with high risk for esophageal SCC compared to low-risk areas. ${ }^{9}$ A meta-analysis showed that HPV16 and 18 can be detected in esophageal SCC. HPV16, but not HPV18, is significantly associated with the risk of esophageal SCC. ${ }^{10}$ Therefore, the objective of the present study was to screen for the presence of High Risk Human Papilloma Viruses 16 and 18 (HR-HPV16\&18) among Sudanese patients with esophageal cancer.

\section{Materials and methods}

The present study investigated 100Sudanese patients with esophageal cancers, 54 were males and 45 were females (male/female ratio, 1.20:1.00), aged between 12 and 98years with mean age of 60 years old, were diagnosed as having esophageal carcinomas, were investigated for the presence of HR-HPV subtypes $16 \& 18$ using C1P5 (HPV16 E6/18 E6 Antibody) immunohistochemistry. Immunohistochemical positive results were further confirmed by Polymerase Chain Reaction (PCR). The diagnosis was based on clinical examination and histological features of the biopsy. Esophageal cancer diagnosis was confirmed base on Royal College of Pathologists criteria (Royal College of Pathologists, 2005). The esophageal cancer including 90/100 (90\%) squamous cell carcinomas (SCCs) and 10/100 (10\%) adenocarcinoma. The sample included full coverage of patients with esophageal lesions referred to Radiation and isotope Centre Khartoum with available sample quantity and data. Ethical consent was obtained from ethical committee of the Faculty Research Board and Hospital.

C1P5 immunohistochemistry (IHC) was performed on formalinfixed paraffin embedded (FFPE) tissue sections using kits from (Dako Agilent Technologies Company). After antigen retrieval, sections were incubated with mouse monoclonal C1P5, and then En Vision System HRP anti-mouse, followed by diaminobenzidine (DAB) chromogen and counterstaining with hematoxylin. Cervical cancer sections known to be HPV-positive were used as a positive control, and omission of primary antibody was used as a negative control. All C1P5 IHC slides were semi quantitatively scored by two investigators for intensity of staining in the cell nucleus and cytoplasm. Intensity was scored as 0 (none), 1(weak), 2 (moderate), or 3 (strong), with 0 or 1 scores defined as negative and 2 or 3 defined as positive. C1P5 scoring was performed without knowledge of HPV infection status. 


\section{Results}

This study investigated 100 tissue samples which were obtained from Sudanese patients with esophageal carcinoma (90 patients were with squamous cell carcinoma and 10 were with adenocarcinoma). Of the 100 study subjects 54 were males and 45 were females their age ranging from 12 to 98 years old with a mean age of 60 years. The majority of the study populations were at the age range 66-75years constituting 26 patients followed by age ranges, both 5665 and $<45$ years representing 24 patients. Moreover, 16 patients were identified among age group 46-55years, hence only 10 patients were found among age group 76+years, as indicated in (Figure 1).

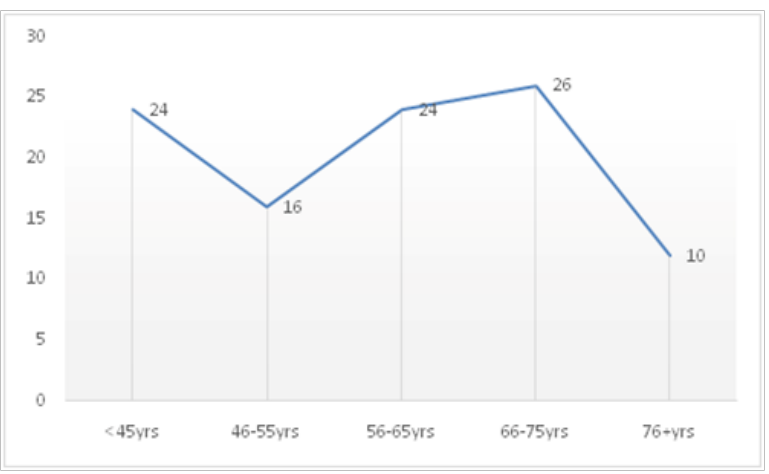

Figure I Description of the study population by age.

However, most of the patients were diagnosed as having Squamous cell carcinoma representing $90 \%$ followed by Adenocarcinoma representing $10 \%$. Of the 90 patients with Squamous cell carcinoma, $52 \%$ were males and $48 \%$ were females. Of the 10 patients with Adenocarcinoma $73 \%$ were males and $27 \%$ were females.

Regarding IHC staining for HPV 16 and 18, positive findings were revealed in 13/100 (13\%) and couldn't be disclosed in $87(87 \%)$ of the study subjects, hence, none of the cases was identified with HPV18 (confirmed by PCR), as shown in Microphotograph $1 \& 2$. The risk associated with HPV 16 as etiological factor for esophageal cancer and the Odd Ratio (RO) and the 95\% confidence interval was 31(1.81$528), \mathrm{P}<0.017$. Of the 54 males with esophageal cancer, $7 / 54(12.9 \%)$ were identified with HPV16 infection and the remaining 47/54(87.1\%) found without HPV16 infection. Of the 45 females with esophageal cancer, 6/45(13.3\%) were found positive for HPV 16 immunostaining and the remaining 39/45(86.7 \%) were found negative for HPV 16.

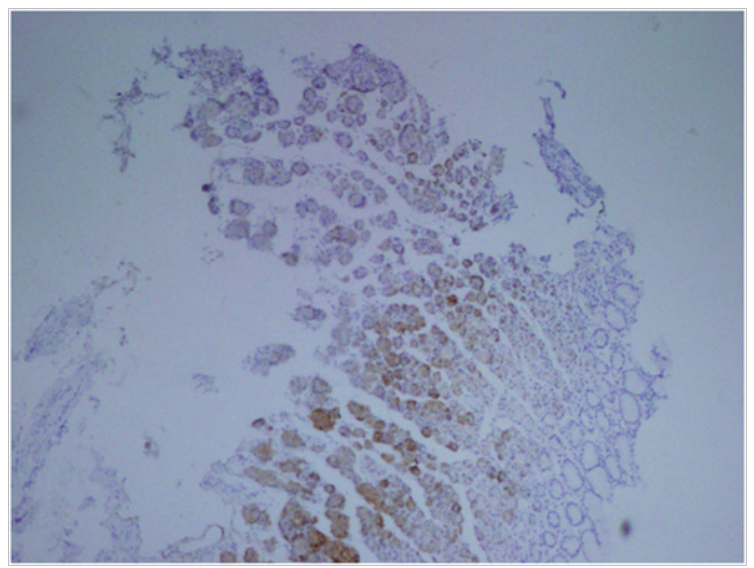

Microphotograph I Esophageal carcinoma showing positive HPVI6 expression (Immunohistochemical staining using CIP5 antibody), En Vision System HRP. XI00.

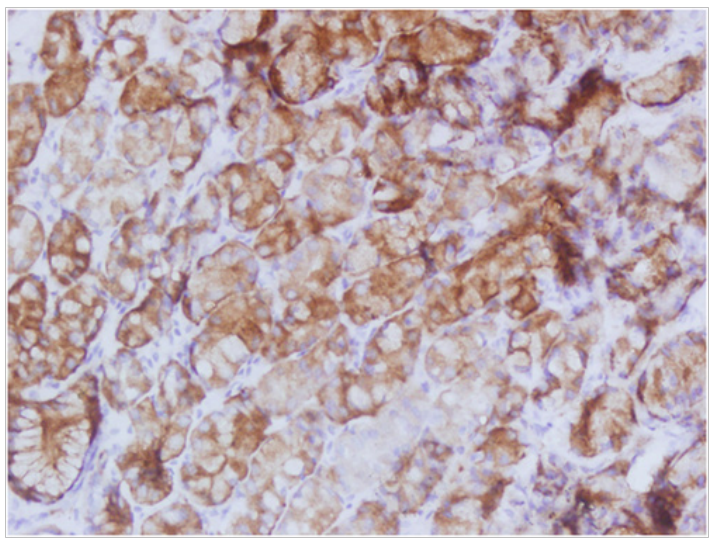

Microphotograph 2 Esophageal carcinoma showing positive HPVI6 expression (Immunohistochemical staining using CIP5 antibody), En Vision System HRP. X400.

The association of HPV 16 risk with sex was OR $(\mathrm{CI})=0.97(0.30$ 3.12), $\mathrm{P}<0.9$.

According to cancer type, of the 90 cases of the Squamous cell carcinoma, 11/90 (12.2\%) were found with positive HPV16 and the remaining 79/90 (87.8\%) were negative. Of the 10 cases of the Adenocarcinoma, 3/10(30\%) were found with positive HPV16 and the remaining $8 / 10(70 \%)$ were negative, as indicated in Table 1 . The association of HPV 16 risk with Adenocarcinoma was OR (CI) = 0.37(0.084-1.56), $\mathrm{P}<0.18$, as indicated in (Table 1) \& ( Figure 2).

Table I Distribution of IHC results of HPV subtypes I6, I8 by sex and cancer type

\begin{tabular}{llll}
\hline Sex & Positive & Negative & Total \\
\hline Males & 7 & 47 & 54 \\
Females & 6 & 39 & 45 \\
Total & 13 & 87 & 100 \\
Cancer Type & & & \\
Squamous & 11 & 79 & 90 \\
Adenocarcinoma & 3 & 7 & 110 \\
Total & 14 & 86 & 100 \\
\hline
\end{tabular}

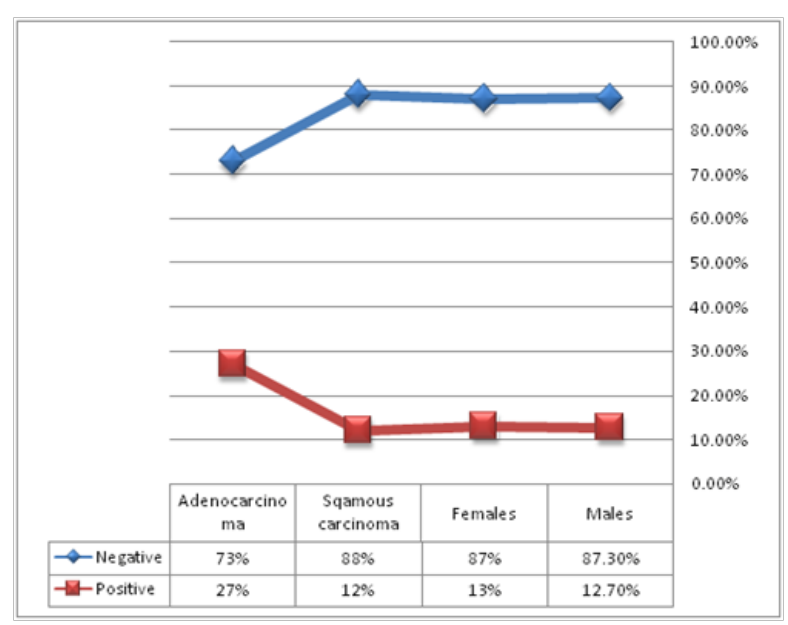

Figure 2 Description of IHC results of HPV subtypes 16,18 by sex and cancer type. 
Concerning IHC staining results of HPV subtypes 16, 18 with age distribution, the highest positive results were found in age group $66-75$ years, representing $6(23 \%)$, followed by $(<45 \& 56-65)$ and $46-55$ constituting $3(12.5 \%)$ and $1(6.3 \%)$ respectively, where's; the groups with the highest negative results were $<45$ and $56-65$ years, representing $21(23.6 \%)$ in each followed by $66-75,46-55$ and $76+$ constituting $20(22.5 \%), 15(16.8 \%)$ and $12(13.5 \%)$ in this order, as shown in (Table 2) \& ( Figure 3).

Table 2 Distribution of IHC results of HPV subtypes 16,18 by age

\begin{tabular}{llll}
\hline Age Group & Positive & Negative & Total \\
\hline$<45$ years & 3 & 21 & 24 \\
$46-55$ & 1 & 15 & 16 \\
$56-65$ & 3 & 21 & 24 \\
$66-75$ & 6 & 20 & 26 \\
$76+$ & 0 & 10 & 12 \\
Total & 13 & 87 & 100
\end{tabular}

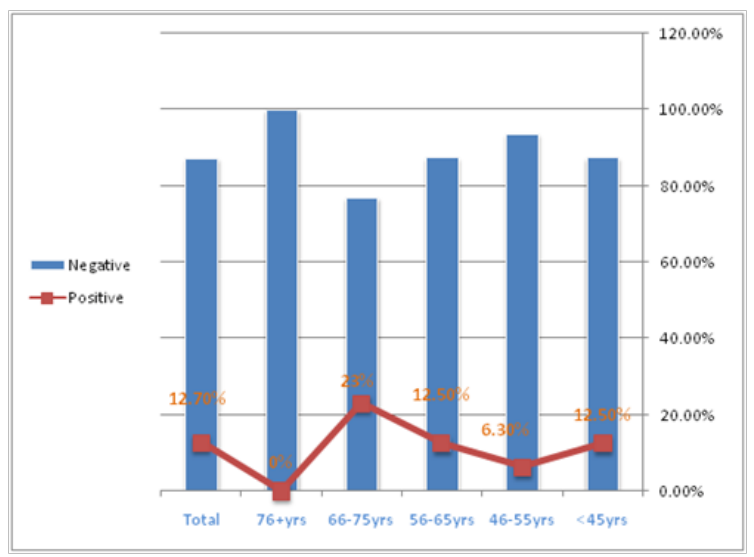

Figure 3 Description of IHC results of HPV subtypes 16,18 by age.

\section{Discussion}

HR-HPVs particularly subtypes 16 and 18 have been linked to the etiology of several cancers including esophageal cancer. However, there is a lack of data regarding the relationship between HPV infection and esophageal carcinoma in the Sudan. Thus the present study is an attempt to explore the role of HPV in the etiology of esophageal cancer and to stimulate further research in this area.

The present study found a prevalence of $13 \%$ of HR-HPV 16 infection among Sudanese patients with esophageal cancer.HPV related esophageal SCC detection rates are greatly variable across different countries. Geographic location likely accounts for a majority of the variation in HPV prevalence, with high-incidence regions including Asia reporting significantly higher HPV related esophageal SCC infection rates compared with low-incidence regions such as Europe, North America, and Oceania. ${ }^{11}$ Studies have shown that Asia is high-risk region. ${ }^{12-14}$ In a meta-analysis study, an overall HPV prevalence of $30.6 \%$ was calculated, but a region-specific infection rate of $10.1 \%$ for Canada and the United States. ${ }^{12}$ There was evidence of an increased risk of esophageal SCC in patients with HPV infection odds ratio (OR) $2 \cdot 69,95 \%$ confidence interval $(\mathrm{CI}) 2 \cdot 05-3 \cdot 54$. The prevalence of HPV in esophageal SCC was found to be $24 \cdot 8 \%$. There was an increased risk associated with HPV-16 infection (OR $2 \cdot 35,95 \%$ CI $1 \cdot 73-3 \cdot 19)$. Subgroup analyses showed geographical variance, with Asia (OR 2.94, 95\% CI 2·16-4.00), and particularly
China (OR 2 $85,95 \%$ CI 2·05-3 96 ) being high-risk areas. ${ }^{14}$

However, we didn't found any literature regarding the relationship between esophageal carcinoma and HPV reported from Sudan. In study to determine the frequency and genotype of human papillomavirus (HPV) infections in head and neck squamous cell carcinomas (HNSCCs), 6 of the 150 (4\%) HNSCCs were HPV positive. HPV16 was the most prevalent type, with single infections present in $3 / 6$ (50\%) cases, whereas HPV18 and HPV33 were detected in 2/6 $33 \%$ ) and $1 / 6(17 \%)$, respectively. HPV infections were detected in $3(50 \%)$ cases of oral cavity and $3(50 \%)$ cases of pharynx. ${ }^{15}$

In the current study the detected cases of HPV infections were infected with HPV16 when further confirmed with molecular tests. Such findings were reported in several studies. In a study investigated 8990 esophageal squamous cell carcinoma (SCC) patients and 174 esophageal adenocarcinomas patients, the prevalence of HPV in esophageal SCC was $22.2 \%$, HPV16 was the most frequently observed subtype with a summarized prevalence of $11.4 \%$. With respect to esophageal adenocarcinoma, HPV prevalence was $35.0 \%$ (95\% CI, 13.2-65.7\%) and HPV-16 prevalence was $11.4 \%$. Significant association was observed between HPV infection and esophageal SCC with a summarized odds ratio of 3.32 (95\% CI, 2.26-4.87). According to HPV16, the strength of the association was found to be $3.52(95 \%$ CI, 2.04-6.07). ${ }^{16}$ Though HPV18 was not detected in the present study but studies have reported low prevalence rates in the highly infected regions. ${ }^{17}$

Although the prevalence of esophageal cancer was higher among males, but the prevalence rates of HPV16 were relatively similar for both sex. However, we didn't come across study showing significant variation in the prevalence of HPV infections in regard to the sex. When calculating the prevalence rate from the same number of sex, it appears greater among females due to the low number of females in regard to the total number of the study population. In the present study we observed variable frequencies among different age ranges, but the HPV infection was more frequent among elder population. The limitations in this study include; it's retrospective setting and the strict detection of only two HR-HPV subtypes. This in addition to the missing of some demographical data. But still it has a value in directing further studies in this context.

\section{Conclusion}

HR-HPV subtype 16 is strongly contributed to the etiology of esophageal carcinoma in Sudan. Urgent preventive strategies are highly recommended to reduce the burden of esophageal cancer in Sudan.

\section{Acknowledgments}

None.

\section{Conflicts of interest}

Authors declare that there is no conflict of interest.

\section{References}

1. Tan C, Qian X, Guan Z, et al. Potential biomarkers for esophageal cancer. Springerplus. 2016;16(5):467.

2. Rustgi AK, El-Serag HB. Esophageal carcinoma. $N$ Engl J Med. 2014;371:2499-2509.

3. Rubenstein JH, Shaheen NJ. Epidemiology, Diagnosis, and Management of Esophageal Adenocarcinoma. Gastroenterology. 2015;149(2):302e1$317 \mathrm{e} 1$. 
4. Alsop BR, Sharma P. Esophageal Cancer. Gastroenterol Clin North Am 2016;45(3):399-412.

5. Chung CS, Lee YC, Wu MS. Prevention strategies for esophageal cancer: Perspectives of the East vs. West. Best Pract Res Clin Gastroenterol. 2015;29(6):869-883.

6. Kuang JJ, Jiang ZM, Chen YX, et al. Smoking Exposure and Surviva .of Patients with Esophagus Cancer: A Systematic Review and MetaAnalysis. Gastroenterol Res Pract. 2016;2016:7682387.

7. Thrift AP. The epidemic of oesophageal carcinoma: Where are we now? Cancer Epidemiol. 2016;41:88-95.

8. Andrici J, Eslick GD. Hot Food and Beverage Consumption and the Risk of Esophageal Cancer: A Meta-Analysis. Am J Prev Med. 2015;49(6):952-960.

9. Xu W, Liu Z, Bao Q, et al. Viruses, Other Pathogenic Microorganisms and Esophageal Cancer. Gastrointest Tumors. 2015;2(1):2-13.

10. Yong F, Xudong N, Lijie T. Human papillomavirus types 16 and 18 in esophagus squamous cell carcinoma: a meta-analysis. Ann Epidemiol. 2013;23(11):726-734

11. Ludmir EB, Stephens SJ, Palta M, et al. Human papillomavirus tumor infection in esophageal squamous cell carcinoma. J Gastrointest Oncol. 2015;6(3):287-295
12. Syrjänen K. Geographic origin is a significant determinant of human papillomavirus prevalence in oesophageal squamous cell carcinoma: systematic review and meta-analysis. Scand J Infect Dis. 2013;45(1):118.

13. Yong F, Xudong N, Lijie T. Human papillomavirus types 16 and 18 in esophagus squamous cell carcinoma: a meta-analysis. Ann Epidemiol. 2013;23(11):726-734

14. Hardefeldt HA, Cox MR, Eslick GD. Association between human papillomavirus (HPV) and oesophageal squamous cell carcinoma: a meta-analysis. Epidemiol Infect. 2014;142(6):1119-1137.

15. Ahmed HG, Mustafa SA, Eltom FM, et al. Frequency and genotype of human papillomavirus among Sudanese patients with head and neck tumors. E cancer medical science. 2012;6:282.

16. Li X, Gao C, Yang Y, et al. Systematic review with meta-analysis: the association between human papillomavirus infection and oesophageal cancer. Aliment Pharmacol Ther. 2014;39(3):270-281.

17. Liu T, Liu Q, Liang M, et al. Viral load of HPV 16/18 in esophageal squamous cell carcinoma in three ethnic groups living in Xinjiang Autonomous Region, China. Mol Biol Rep. 2013;40(2):2045-2052. 